\title{
Review of: "Long-term Condition Management for Prisoners: exploring prevalence and compliance with national monitoring processes"
}

\author{
Jake $\operatorname{Hard}^{1}$ \\ 1 National Health Service
}

Potential competing interests: The author(s) declared that no potential competing interests exist.

The impact of the Quality Outcomes Framework has been well-recognised for community General Practice for many years. This paper provides a clear account for the need to improve the measurement and monitoring of the health of people in prison utilising these standards in order to help achieve equivalent healthcare. The paper is presented at an important moment given the planned rollout of GP registration for people arriving in prison in the near future. 